\title{
Monitoring and Research on Temperature Field and Juncture Deformation of CRTS II Slab BallastlessTrack in High Temperature
}

\author{
Hongyao $\mathrm{Lu}^{1, \mathrm{a}}$ and Yuelei $\mathrm{He}^{1, \mathrm{~b}}$ \\ ${ }^{1}$ School of Urban Railway Transportation,Shanghai University of Engineer Science,Shanghai \\ 201620, China \\ alhy@sues.edu.cn, bhyldoc@163.com
}

\begin{abstract}
Keywords:Slab ballastless track, Juncture defects, Monitoring technology,Temperature load, Relative displacement.
\end{abstract}

\begin{abstract}
Aiming at the juncture defects of CRTS II slab ballastless track in China high speed railway, an on-line monitoring system for temperature field and deformation of track slab was set up.The temperature variation and the relative displacement between slabs and junctures were obtained in real time. Experiments showed that the relative displacement of wide and narrow juncture could reach $0.11 \mathrm{~mm}$ and $0.70 \mathrm{~mm}$ caused by the temperature change of the track slab, which was the main reason for junctures'destruction. The linear correlation coefficients between the relative displacement and the temperature of the junctureswere 0.870 and 0.946 , which could provide references for maintenance and repair of ballastless track.
\end{abstract}

\section{Introduction}

CRTS slab ballastless track is widely used in China high speed railway because of its high stability and homogeneous support stiffness. The slabs are joined by longitudinal prestressed reinforcements, which determines that they are more sensitive to changes in temperature. Especially in the high temperature, the internal temperature is higher than the longitudinal locking temperature. The track slab is subjected to compressive stress in summer,which is one of the main causes of disease in junctures.In the actual field investigation, it is found that there are obvious separation and damagein the junctures.

As the key parts connecting track slabs, the junctures' damage could weaken the track slabs vertical connection and affect track irregularity,which is a direct threat to the traffic safety.The relevant scholars carried out a lot of research work. The interface crack between slab and juncture of slab ballastless track is investigated,and causes and harm of juncture cracking are analyzed[1].Aiming at the temperature field of track slab,an effective and accurate prediction of the high-speed railway ballastless track structure temperature field were established[2]. Based on the finite element theory, a three dimensional model of CRTS II slab ballastless track on long temperature span concrete bridge was established to analyze the mechanical properties of its structure and the adaptability of the design load combination of base slab [3]. At the same time, he calculation model of CRTS type II slab track on bridge is established, and the influence of wide juncture cracking on mechanical behavior of CRTS type II slab track under different loads is analyzed[4].Many researchers have been committed tothe research on temperature field and juncture deformation of CRTS II slab ballastless track.

According to the actual operation situation of CRTS II slab ballastless track in East China, this paper selects a broken juncture as the object of study and bulid an intelligent track board key parameter monitoring terminal. By means of field measurement, the relative displacement of the junctures and the temperature field of the track slab were studied. The change rule of relative displacement value and temperature load in the juncture is analyzed. The law of relative displacement and temperature change in the juncture is fitted and analyzed,which could provide reference for the relevant standards of high-speed railway maintenance and repair. 


\section{Establishof monitoring system}

In view of the actual maintenance conditions and operation requirements, the track slab temperature field and deformation testing equipment was established to obtain real-time and on-line monitoring key parameters of the CRTS type II track slab. The overall framework of the system as shown in Figure 1, andthe key technologies include as follows:

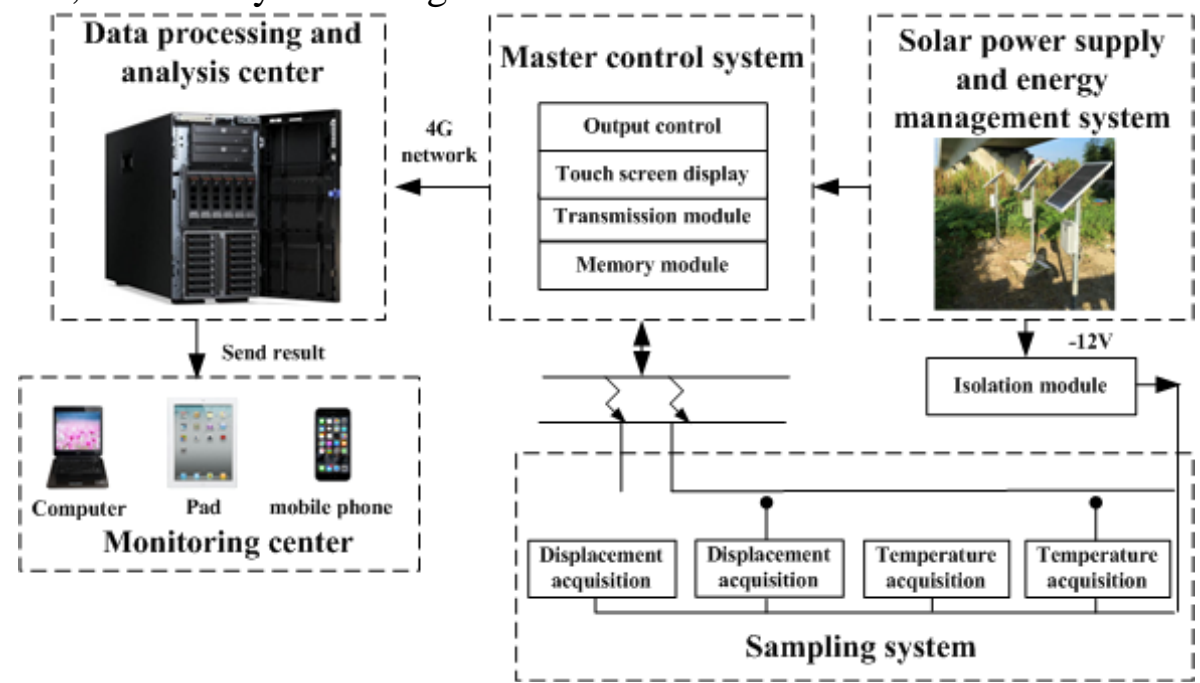

Fig.1.Test system framework

(1)Fuzzy control solar energy management system

According to the operating mechanism and working characteristics of power supply load, a multi-level fuzzy control strategy is proposed. Firstly, the maximum power point tracking control strategy based on fuzzy teaching and learning is adopted in the solar panel, which takes into account both the solar maximum power point tracking control and the solar energy tracking control. At the same time, the charging and discharging management of the energy storage unit is realized, and the efficiency of the solar power generation is effectively improved. Secondly, aiming at the detection characteristics of various sensors, multi-stage power supply mode is adopted. The monitoring power consumption is reduced, and the long-term on-line monitoring of the intelligent terminal is ensured.

(2) High precision data acquisition intelligent test system

The collected data is sent to the monitoring center in real time through the 4G network, and the data processing and analysis process is realized in the back-end processing and analysis center by system software;Users use terminal devices to achieve the setting of various test parameters and starting or stopping instructions. The system automatically completes the processing and analysis of large amount of datas, and returns the processing result to the user terminal.

\subsection{Sensors installation}

The test selection time is from July 22, 2016 to September 14, 2016, corresponding to the high temperature weather in summer.The monitoring time described in the later paragraph is the period of time.The selected track slab has an obvious drop in the juncture.The crack gauges are respectively arranged at the positions of wide and narrow junctures, which has the distance of the $50 \mathrm{~mm}$ and $180 \mathrm{~mm}$ on the upper surface of the track slab to monitor the relative displacement between the track slab and the juncture concrete.The layout of the cracks does not produce the output under the train load,only the output occurs when the concrete surface produces strain at the tested juncture. When the crack gauge is pulled, the displacement output is positive, and the displacement output is negative when it is pressed.Its sampling accuracy is $0.01 \mathrm{~mm}$, and the sampling frequency is half an hour.

The temperature sensors were punched in the middle and the end of the track slab inside respectively along the central line, which monitors temperature at different locations within the track slab. The temperature sensors in different holes are arranged at the $50 \mathrm{~mm}$ and $180 \mathrm{~mm}$ on the upper surface of the board respectively. When analyzing the whole temperature at the depth, the average 
temperature at the same depth in the two hole of the slab is taken as the whole temperature of the depth.Its sampling accuracy is $0.01^{\circ} \mathrm{C}$, and the sampling frequency is half an hour.

\section{Monitoring data analysis}

\subsection{Integral statistical law}

The relative displacements at wide and narrow junctures were monitored, and the whole temperature law of different depths at different depths on the track plate was obtained simultaneously in the monitoring time.The corresponding relation between relative displacement and temperature is shown in Figure 2.The greater the temperature difference between the juncture temperature and the crack gauge locking temperature, the greater the relative displacement at the juncture.

The measured datas show that the temperature variation of the narrow juncture is $11.64^{\circ} \mathrm{C}$ and the maximum relative displacement at narrow juncture reaches $0.67 \mathrm{~mm}$; The temperature variation at the wide juncture is $14.04{ }^{\circ} \mathrm{C}$, and the maximum relative displacement reaches $0.09 \mathrm{~mm}$ when compared with the locking time.

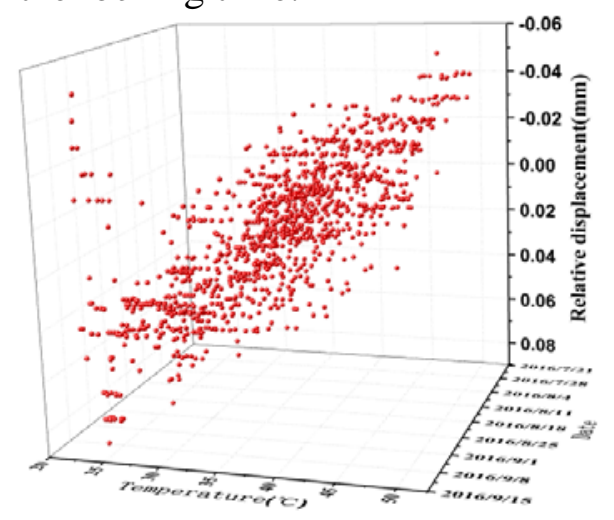

(a) Wide juncture

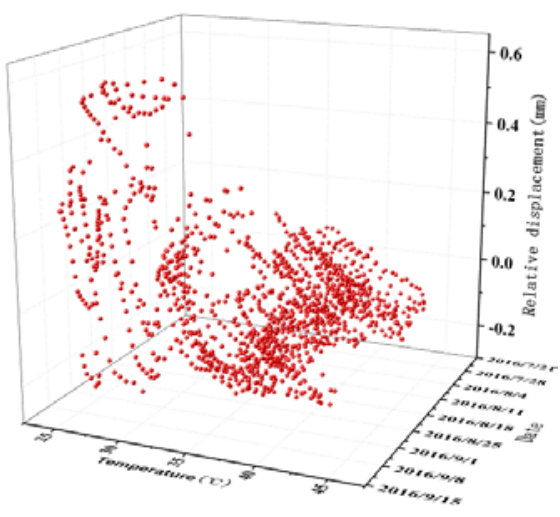

(b) Narrow juncture

Fig.2. Relationship between displacement and temperature variation

It can be seen that the temperature amplitude of wide juncture layer is larger than that of narrow juncture during monitoring time.The main reason is that the wide juncture position is closer to the surface of track slab,and the influence of meteorological parameters such as solar radiation and wind load are greater.The heat exchange is more obvious, and the whole temperature change amplitude is greater.

Although the whole temperature variation amplitude at the narrow juncture is smaller than that of the wide juncture, the relative displacement amplitude at the narrow juncture is larger than that at the wide juncture during monitoring. This is mainly caused by the structural characteristics of the track slab at design time:To weaken the influence of track slab thermal expansion and contraction,some38mmdepth prefabricated gaps are set on the top surface of the track board.The prefabricated gaps divided the upper surface of the slab track into discrete "broad sleeper" units,so the relative displacement amplitude of the wide juncture under the temperature load is less than the value at the narrow juncture.It is also proved that the disease in the narrow juncture position is more obvious in the actual investigation.

\subsection{Dailyrelative displacement amplitude of juncture}

During the monitoring time,the daily relative displacement amplitude in the wide juncture reaches $0.11 \mathrm{~mm}$, and the daily relative displacement in narrow juncture reaches $0.70 \mathrm{~mm}$, as shown in Figure 3. 


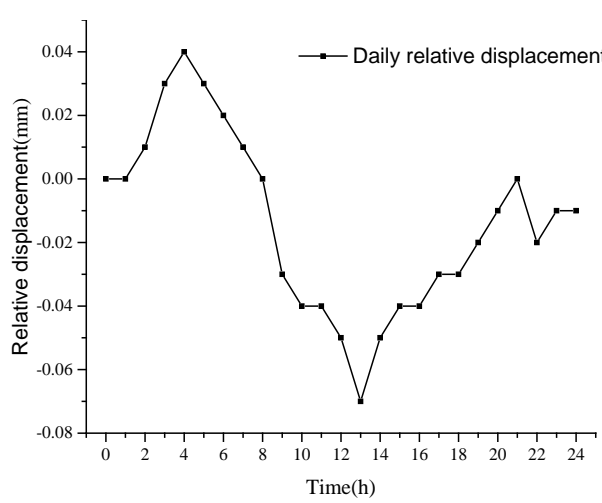

(a) Wide juncture

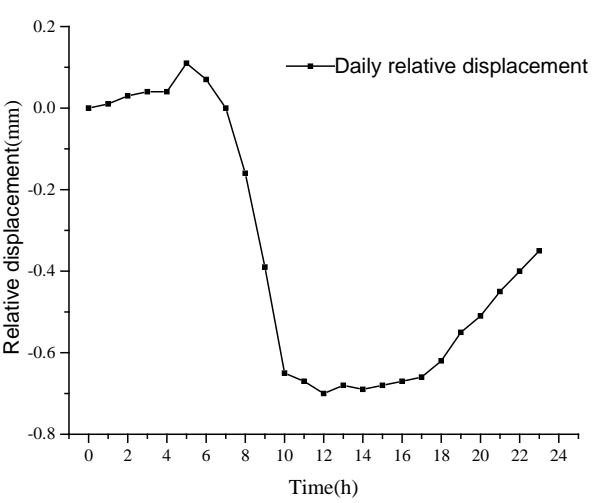

(b) Narrow juncture

Fig.3. Daily relative displacement amplitude of junctures

The maximum daily relative displacement amplitude of junctures appears mostly from 3 to 5 a.m.. Under the action of wind load, the whole temperature of slab decreases to the minimum value, and the tensile value is reached at the juncture;The minimum daily relative displacement amplitude of junctures appears mostly from 12 to 14 noon. Under the action of solar radiation, the whole temperature of the track slab is increased to the maximum value, and the juncture reaches the compression extremum.

\subsection{Relation between relative displacement of junctures and temperature load}

The whole temperature change of track slab directly affects the longitudinal deformation of track slab. If the failure limit of juncture concrete is reached, it will seriously affect the safety of the line.

In order to analyze the rule of the relative displacement and whole temperature at the juncture,the temperature difference between the daily extreme temperature and the lock time of the crack gauge is calculated, and the relative displacement at the corresponding juncture is recorded respectively. The linear regression of the temperature difference at the locking moment and the relative displacement of the juncture is carried out.Ninety sets of sample datas are selected as the fitting samples, and it is found that the relative displacement of the juncture in the high temperature weather is basically linear with the change of the whole temperature.The fitting relation between the relative displacement and the whole temperature of the juncture is shown in Figure 4.

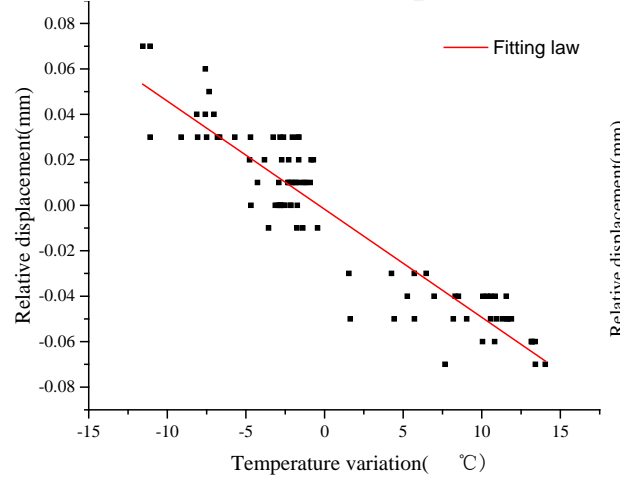

(a) Wide juncture

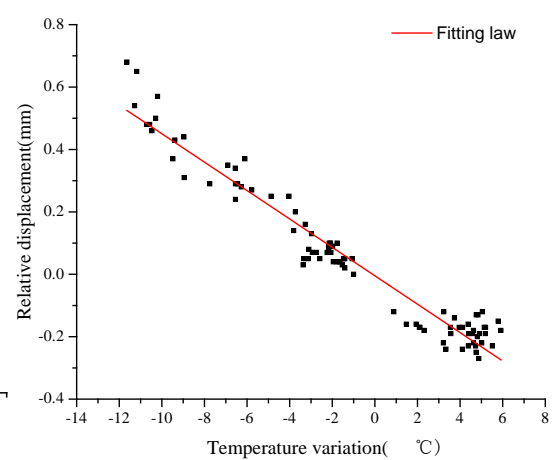

(b) Narrow juncture

Fig.4. Fitting law of relative displacement and temperature variation

In the type: $\Delta X_{\mathrm{k}}$ is the relative displacement at wide juncture; $\Delta X_{\mathrm{z}}$ is the relative displacement at narrow juncture; $\Delta T_{\mathrm{k}}$ is the temperature variation at wide juncture; $\Delta T_{\mathrm{z}}$ is the temperature variation at narrow juncture. From the laws of rotation we can find:

$$
\begin{aligned}
& \Delta X_{\mathrm{k}}=-4.76 \times 10^{-3} \times \Delta T_{\mathrm{k}}-1.73 \times 10^{-3} \\
& \Delta X_{\mathrm{z}}=-4.55 \times 10^{-2} \times \Delta T_{\mathrm{z}}-6.23 \times 10^{-3}
\end{aligned}
$$

The linear fitting correlation coefficient for the relative displacement at wide junctures with temperature changesis 0.870 , and the linear correlation coefficient between the relative 
displacements at narrow junctures and the temperature changes is 0.946 .

Analysis shows that the relative displacement at the narrow juncture is larger than the relative displacement at the wide juncture under the same temperature variation. The width of the juncture is close to the upper surface of the track, and the law of heat transfer between layers is more complicated. Compared with the fitting rule at narrow juncture, the linear correlation coefficient between relative displacement and temperature is smaller. To achieve higher precision fitting, it is necessary to increase the monitoring of the environmental parameters such as solar radiation and wind speed which closely affect the surface temperature of the slab.

\section{Conclusion}

Aiming at the juncture defects of CRTS II slab ballastless track in China high speed railway,this paper investigated the influence of the whole temperature load on juncture defects based on measured datas. The main conclusions are as follows.

(1)An intelligent track slab temperature field and relative displacement on-line monitoring system is set up. Sensors are used to study the relative displacement of track slab junctures under temperature load. The greater the temperature difference between the junctures temperature and the locking temperature, the greater the relative displacement of the juncture occurs;

(2)The maximum relative displacement of wide and narrow junctures reached $0.11 \mathrm{~mm}$ and $0.70 \mathrm{~mm}$ respectively in the high temperature weather. The relative displacement at the narrow juncture is larger than that in the wide juncture, and it is also verified by the actual investigation that the disease in the narrow juncture is more serious;

(3)Based on the measured datas, the linear regression analysis model of track slab temperature and relative displacementis are established,and the correlation coefficients are 0.870 and 0.946 respectively. The relative displacement of the juncture can be predicted preliminarily based on the change of temperature, which can provide reference for the maintenance and repair works.

\section{Acknowledgement}

In this paper, the research was sponsored by the Nature Science Foundation (Project No.51478258) and Key Technology R\&D Project of Shanghai Committee of Science and Technology (Project No. 16030501400) and the research and development of science and technology of Shanghai Railway Burea (Project No. 20160285).

\section{References}

[1] LOU Liangwei, XIE Yongjiang, XIN Xuezhong, ZHONG Xinhua. Study on crack in interface between track slab and wide joint for CRTS II slab-type ballastless track [J].Railway Engineering , 2015(01): 98-101.

[2] OU Zu-min, SUN Lu. Simplified Calculation Method on Nonlinear Temperature Effects of High-speed Railway Ballastless Track Slab [J]. Journal of The China Railway Society, 2015(06): 79-87.

[3] LI Dongsheng, NIU Bin,HU Suoting,DONG Liang. Mechanical Properties of CRTS II slab ballastless track on long Temperature Span Concrete Bridge [J]. China Railway Science, 2016(03): 22-29.

[4] ZHAO Lin, LIU Xueyi, ZHAO Huawei, DONG Jiajia. The study of influence on track stress caused by the cracking at wide juncture of CRTS II prefabricated slab track [J]. Journal of Railway Science and Engineering, 2016(01): 9-14. 